\title{
Hidden variable interpretation of spontaneous localization theory
}

\author{
Daniel J. Bedingham*†
}

June 24, 2018

\begin{abstract}
The spontaneous localization theory of Ghirardi, Rimini, and Weber (GRW) is a theory in which wavepacket reduction is treated as a genuine physical process. Here it is shown that the mathematical formalism of GRW can be given an interpretation in terms of an evolving distribution of particles on configuration space similar to Bohmian mechanics (BM). The GRW wavefunction acts as a pilot wave for the set of particles. In addition, a continuous stream of noisy information concerning the precise whereabouts of the particles must be specified. Nonlinear filtering techniques are used to determine the dynamics of the distribution of particles conditional on this noisy information and consistency with the GRW wavefunction dynamics is demonstrated. Viewing this development as a hybrid BM-GRW theory, it is argued that, besides helping to clarify the relationship between the GRW theory and BM, its merits make it worth considering in its own right.
\end{abstract}

\section{Introduction}

The failings of standard quantum mechanics (SQM) are best exemplified with the problem of quantum measurement: In SQM the two rules for the timeevolution of the wavefunction of a system (the Schrödinger equation and the reduction postulate) require a fundamental distinction between processes that are measurements and those that are not; since the concept of measurement is vague and ill-defined it follows that the theory is vague and ill-defined.

Whilst many are content to avoid this embarrassing problem by disregarding wavepacket reduction and taking the view that the Schrödinger equation gives the complete picture, this leads unavoidably to the existence of macroscopic superposition states from which there is no indication of how to obtain the definite world of our experience. The situation is concisely summed up by John Bell [1: Either the wavefunction, as given by the Schrödinger equation, is not everything, or it is not right.

\footnotetext{
* Blackett Laboratory, Imperial College, London SW7 2BZ, UK.

†email: d.bedingham@imperial.ac.uk
} 
This statement suggests two possible approaches to dealing with concerns over quantum theory. The first approach is to include additional state variables (hidden variables) and is well illustrated by Bohmian mechanics (BM) (also known as de Broglie-Bohm pilot wave theory) 22. The second approach is to replace the Schrödinger equation with a more general stochastic equation capable of describing both unitary behavior and random wavefunction collapse events (making no reference to the concept of measurement). This approach is known as dynamical reduction (DR) [3, 4. (Note that in SQM we also make the assumption that the Schrödinger equation is not universally valid although the specification is ill-defined.)

In BM the particle positions are definite possessed properties of the system under consideration. The wavefunction $\psi$, which satisfies the Schrödinger equation at all times, is viewed as a pilot wave whose role is to guide the trajectories of the particles. The flow is such that the forward equation (or continuity equation) for the probability distribution of particles on configuration space is identical to the equation describing the time-evolution of $|\psi|^{2}$. Therefore, in the case that the probability distribution of particles on configuration space is equal to $|\psi|^{2}$ at some initial point in time, it will also be the case at any later time. This condition, known as quantum equilibrium, is a subtle issue. It is necessary in order for BM to reproduce quantum predictions (for example, it will guarantee that classically expected particle positions will equate to quantum expectations of position operators), however, it must be broken in general as the particles map out definite trajectories which determine such things as quantum measurement outcome: 1 .

Conversely, in DR, the wavefunction describes the complete state but satisfies a stochastic generalization of the Schrödinger equation in which collapses occur randomly. This happens in such a way that for superpositions involving large numbers of particles the wavefunction collapse naturally occurs very rapidly (and with the correct quantum probability), whilst for small numbers of particles the effects are negligible. The theory is interpreted either by treating the wavefunction as representative of matter density in the world, or by forming (an approximate) classical image of the world from the classical stochastic inputs to the model (e.g. the discrete random collapse centers). Here we shall focus on the spontaneous localization theory of Ghirardi, Rimini, and Weber (GRW) [5], in which the random collapses occur in a particle position-state basis. The privileged role of the particle positions in the GRW theory draws parallels with $\mathrm{BM}$, but overall, the two approaches are quite different.

However, the aim of this paper is to show that the mathematical formalism of GRW theory can be given an explanation in terms of an evolving distribution of particle positions on configuration space. On one hand this is a novel interpretation of the theory; on the other hand this is a new BM-GRW hybrid theory employing both additional state variables and modified wavefunction dynamics (this is the position taken in ref. [6] where such a BM-GRW hybrid theory is

\footnotetext{
${ }^{1}$ Quantum equilibrium is only true in an effective sense and relies on arguments involving decoherence to determine the effective wavefunction to which it applies at any stage.
} 
proposed). In this sense it is based on a third possibility besides the two given above by Bell, that the Schrödinger wavefunction is neither right nor is it everything. By constructing such a theory it is possible to get the individual benefits both of BM (a clear ontological meaning in terms of definite particle positions making it a relatively straightforward business to interpret the theory), and DR (a wavefunction whose dynamics reflects the particle trajectories).

In GRW theory, $\psi$ and therefore $|\psi|^{2}$ exhibit stochastic behavior. If the wavefunction is to act as the pilot wave for a set of particles then, as with BM, we should expect that a quantum equilibrium condition will be preserved by the dynamics. In order to show this we must demonstrate a stochastic behavior in the distribution of particles on configuration space equivalent to that of $|\psi|^{2}$. We will find that this is achieved by introducing a noisy 'information' process relating to the true positions of the particles. The distribution of particles on configuration space is updated in the manner of Bayesian inference whereby the initial distribution represents the prior and the noisy information process constitutes an acquired stream of evidence. By conditioning on this noisy information we will demonstrate that the usual particle guiding equation of BM produces a stochastic equation for the probability distribution of particles on configuration space which is equivalent to the GRW equation for $|\psi|^{2}$. In fact we will derive the stochastic equation for the wavefunction of GRW theory from the standard Bohmian picture + noisy 'information'.

The idea that the dynamically collapsing wavefunction can be understood in terms of a Bayesian updating procedure has been proposed by Brody and Hughston [7] who considered a discrete basis of energy eigenstates. Starting with a DR model in which the random collapses occur in this energy state basis it was shown that the model could be solved by treating the terminal energy eigenvalue as a hidden variable whose value is gradually revealed by an appropriately defined noisy information process. Nonlinear filtering was used to determine the best estimate of the terminal energy eigenvalue (given the noisy information) and this was shown to be equivalent to the quantum energy expectation. The same authors, along with Macrina, went on to apply this technology to solve a model describing the behavior of financial assets $[8$. (See also [9] for use of nonlinear filtering in solving a DR model describing a simplified EPR experiment.) Here we will generalize this idea to cover a continuous and dynamical hidden variable process (the underlying particle dynamics of BM) and apply it to a realistic DR model, namely the GRW model.

The structure of the paper is as follows. In sections 2 and 3 we briefly outline the mathematical formalisms of BM and the GRW theory respectively. In section 4 we present the nonlinear filtering problem from the innovations approach. This concerns the estimation of an unobserved process given observations of a related process. After demonstrating that the equations of the GRW theory can be rederived from a nonlinear filtering perspective we shall discuss the meaning of what have shown in section 5 . 


\section{Bohmian mechanics}

Consider a quantum system describing a set of particles whose wavefunction $\psi\left(x_{1}, x_{2}, \cdots ; t\right)$ satisfies the Schrödinger equation

$$
i \frac{\partial \psi}{\partial t}=H \psi
$$

The Hamiltonian takes the form $H=-\sum_{i}\left(1 / 2 m_{i}\right) \nabla_{i}^{2}+V$, where $m_{i}$ is the mass of particle $i$ and $V$ is the potential. For notational simplicity we shall use the definitions

$$
\boldsymbol{\nabla}=\left(\nabla_{1}, \nabla_{2}, \cdots\right)^{\mathrm{T}} ; \quad \mathbf{x}=\left(x_{1}, x_{2}, \cdots\right)^{\mathrm{T}} ; \quad \mathbf{M}=\operatorname{diag}\left(m_{1}, m_{2}, \cdots\right) .
$$

By writing the wavefunction as $\psi=R \exp \{i S\}$ we can decompose (11) into the two equations

$$
\frac{\partial S}{\partial t}=-\frac{1}{2}(\nabla S) \cdot \mathbf{M}^{-1} \cdot(\nabla S)+V-\frac{\left(\boldsymbol{\nabla} \cdot \mathbf{M}^{-1} \cdot \nabla R\right)}{2 R},
$$

and

$$
\frac{\partial R}{\partial t}=-(\nabla R) \cdot \mathbf{M}^{-1} \cdot(\nabla S)-\frac{1}{2} R\left(\nabla \cdot \mathbf{M}^{-1} \cdot \nabla S\right) .
$$

Then defining $\rho(\mathbf{x} ; t)=R^{2}=|\psi|^{2}$ we can replace the second of these equations with the forward equation for the quantum probability distribution

$$
\frac{\partial \rho}{\partial t}+\nabla \cdot\left(\rho \mathbf{M}^{-1} \cdot \nabla S\right)=0
$$

Equations (3) and (5) together are equivalent to the Schrödinger equation; the last few lines are nothing more than a rephrasing exercise.

In $\mathrm{BM}$ it is assumed that a complete specification of the state of a system includes not only the wavefunction but also the positions of all the particles that the state is supposed to be describing. Consider a vector set of classical particle trajectories $\mathbf{X}_{t}$ and suppose that the velocities of the particles are given by the guiding equation

$$
\mathbf{V}_{t}=\frac{d \mathbf{X}_{t}}{d t}=\mathbf{M}^{-1} \cdot \nabla S\left(\mathbf{X}_{t}\right) .
$$

This equation expresses the Bohmian particle dynamics. Given this flow, it immediately follows that the probability distribution for the particle positions on configuration space will satisfy a classical forward equation precisely of the form (5). This means that if the probability distribution for the particle positions is equal to $\rho$ at some initial time (quantum equilibrium hypothesis) then it will be equal to $\rho$ at all future times (principle of equivariance). This property ensures that the (classical) statistical properties of the particle positions $\mathbf{X}_{t}$ will be equivalent to the quantum statistics of the position operator $\mathbf{x}$ (at time $t$ ). 
Furthermore we can interpret $S$ as the action for the system of particles and (3) as the Hamilton-Jacobi equation where quantum effects are attributed to the peculiar quantum potential: $-\left(\boldsymbol{\nabla} \cdot \mathbf{M}^{-1} \cdot \boldsymbol{\nabla} R\right) /(2 R)$. At this point we might wish to discard the wavefunction altogether and attempt to take a purely classical view. However, to do this would imply that the quantum potential would have the unusual effect of causing the overall probability distribution of particles $\rho$ to influence the dynamics of individual particles. For this reason the role of $\rho$ must be elevated from epistemical to physical. The wavefunction is therefore retained and treated as a physical 'pilot wave' guiding the flow of particles. It should also be noted that the wavefunction has dynamical degrees of freedom such as spin (not considered here), that cannot be accounted for in terms of particle positions alone. These features require a physical wavefunction.

The advantages that BM has over SQM are that it is well defined and that it offers a clear interpretational framework; there is no doubt as to the meaning of the theory - it concerns the motion of a set of particles.

\section{GRW theory}

Now we turn to the spontaneous localization theory of GRW [5] where it is assumed that the wavefunction gives a complete description of the state of a system. The wavefunction does not satisfy the Schrödinger equation. Instead it satisfies a more general stochastic dynamics which can be approximated by either the Schödinger equation or quantum state reduction in situations where either of those descriptions are appropriate.

Specifically, the Schrödinger equation is supplemented with random spontaneous localization events. Consider a quantum system describing a set of distinguishable particles. (Here, we use the term 'particles' not in the classical sense, as with BM, but simply for convenience in describing a quantum system.) Associated with each particle is a random sequence of Poisson distributed points in time with mass-dependent frequency $\lambda_{i}=\left(m_{i} / m\right) \lambda$, where $\lambda$ is a reference frequency and $m$ is a reference mass. Whenever one of these random times is encountered the wavefunction ceases for an instant to satisfy the Schrödinger equation and undergoes a discrete change. For particle $i$ this is described by

$$
\psi\left(x_{1}, x_{2}, \cdots ; t\right) \rightarrow \psi\left(x_{1}, x_{2}, \cdots ; t+\right)=L\left(x_{i}-z_{i}\right) \psi\left(x_{1}, x_{2}, \cdots ; t\right),
$$

where $L$ is the localization operator. This is given by

$$
L\left(x_{i}-z_{i}\right)=\exp \left\{-\frac{\left(x_{i}-z_{i}\right)^{2}}{2 \sigma^{2}}\right\} .
$$

Here $z_{i}$ is a random variable representing a preferred position in space and $\sigma$ represents the width of the (three-dimensional) Gaussian peak. The effect of the localization operator is to focus the quantum amplitude in configuration space about the point $x_{i}=z_{i}$. The result is a well $x_{i}$-localized wavefunction. 
The probability distribution for the location $z_{i}$ is given by

$$
\mathbb{P}\left(z_{i} \in D_{i}\right) \propto \int_{D_{i}} d z_{i}\left\langle L^{2}\left(x_{i}-z_{i}\right)\right\rangle_{t},
$$

for some region $D_{i}$ of $x_{i}$ values, where we have used $\langle O\rangle_{t}=\int d \mathbf{x} \psi^{*}(\mathbf{x} ; t) O \psi(\mathbf{x} ; t)$ to denote the quantum expectation of operator $O$. This probability rule essentially entails that the localization center is more likely to be where the quantum amplitude is greater.

For the model to work the average effect of localization on the wavefunction of a single particle must be mild, otherwise, quantum interference effects would be lost. Even with this constraint, it follows that for bulk superposition states the wavefunction can be subject to a rapid collapse. The sheer number of particles, each subject to mild collapse effects, along with entanglements between different particle position states, leads to an amplification mechanism. For example, if we take both $\lambda_{i}$ and $\sigma$ to be small $\left(\sim 10^{-17} \mathrm{~s}^{-1}\right.$ and $\sim 10^{-7} \mathrm{~m}$ respectively [5]) then, for an individual particle, localization events are rare but effective. Consequently, the chance of an individual particle undergoing a localization in a given small period of time can be considered to be extremely small. However, with a bulk superposition involving of order $10^{24}$ particles, the chance of at least one constituent particle undergoing a localization in a small time frame is large and the superposition as a whole is suppressed.

The GRW theory as outlined here is a discrete theory of localization events. We can take the continuum limit by letting the frequency of localizations go to infinity such that

$$
\lambda \rightarrow \infty \quad ; \quad \sigma \rightarrow \infty \quad ; \quad \frac{2 \lambda}{\sigma^{2}}=g^{2}
$$

for some constant $g$. It is in fact found that many key effects of the GRW model depend only the combination of factors $g$ and not separately on $\sigma$ and $\lambda[3$. The result of taking this limit is the self contained stochastic differential equation

$$
\begin{aligned}
d \psi= & -i H \psi d t \\
& -\frac{1}{8}\left\{\mathbf{x}-\langle\mathbf{x}\rangle_{t}\right\} \cdot \mathbf{G}^{2} \cdot\left\{\mathbf{x}-\langle\mathbf{x}\rangle_{t}\right\} \psi d t+\frac{1}{2}\left\{\mathbf{x}-\langle\mathbf{x}\rangle_{t}\right\} \cdot \mathbf{G} \cdot \psi d \mathbf{W}_{t},
\end{aligned}
$$

where $\mathbf{G}=g \sqrt{\mathbf{M} / m}$, and $\left\{\mathbf{W}_{t}\right\}$ is a multivariate $\mathbb{P}$-Brownian motion whose components $W_{i ; t}$ satisfy $\mathbb{E}\left[d W_{i ; t}\right]=0$ and $d W_{i ; t} d W_{j ; t}=\delta_{i j} d t$ (E denotes expectation under the measure $\mathbb{P} / 2$. This equation incorporates both the wavefunction

\footnotetext{
${ }^{2}$ To take the limit given by equation (10) we write $\lambda_{i}^{-1}=d t$ and $z_{i}=g_{i}^{-1}\left(d W^{\prime}{ }_{i} / d t\right)$ where $g_{i}=g \sqrt{m_{i} / m} ; W_{i}^{\prime}$ is a standard (base measure) Brownian motion and $d W^{\prime}{ }_{i} / d t$ corresponds to white noise. The white noise scaling factor $g_{i}^{-1}$ will ensure normalizability of $\mathbb{P}$-measure probabilities (9). Then from equation (8) we have

$$
L\left(x_{i}-z_{i}\right)=\exp \left\{\frac{g_{i}^{2} d t}{4}\left(x_{i}-g_{i}^{-1} \frac{d W_{i}^{\prime}}{d t}\right)^{2}\right\} \propto 1-\frac{1}{8} g_{i}^{2} x_{i}^{2} d t+\frac{1}{2} g_{i} x_{i} d W_{i}^{\prime} .
$$
}

The change of measure (from base measure to $\mathbb{P}$ ) defined by (9) enables us to specify a $\mathbb{P}$ - 
dynamics and the probability rule (9). From now on we shall use equation (11) as our expression of the GRW theory.

Writing the wavefunction as $\psi=R \exp \{i S\}$ it is straightforward to show that (11) is equivalent to the following two equations:

$$
\frac{\partial S}{\partial t}=-\frac{1}{2}(\nabla S) \cdot \mathbf{M}^{-1} \cdot(\nabla S)+V-\frac{\left(\boldsymbol{\nabla} \cdot \mathbf{M}^{-1} \cdot \nabla R\right)}{2 R},
$$

and

$$
\begin{aligned}
d R= & -(\boldsymbol{\nabla} R) \cdot \mathbf{M}^{-1} \cdot(\boldsymbol{\nabla} S) d t-\frac{1}{2} R\left(\boldsymbol{\nabla} \cdot \mathbf{M}^{-1} \cdot \boldsymbol{\nabla} S\right) d t \\
& -\frac{1}{8}\left\{\mathbf{x}-\langle\mathbf{x}\rangle_{t}\right\} \cdot \mathbf{G}^{2} \cdot\left\{\mathbf{x}-\langle\mathbf{x}\rangle_{t}\right\} R d t+\frac{1}{2}\left\{\mathbf{x}-\langle\mathbf{x}\rangle_{t}\right\} \cdot \mathbf{G} \cdot R d \mathbf{W}_{t} .
\end{aligned}
$$

Defining $\rho=R^{2}$ and using $d \rho=2 R d R+(d R)^{2}$ (from Itô's lemma), the second of these equation can be used to derive the forward equation for the quantum probability distribution

$$
d \rho=-\nabla \cdot\left(\rho \mathbf{M}^{-1} \cdot \nabla S\right) d t+\rho\left\{\mathbf{x}-\langle\mathbf{x}\rangle_{t}\right\} \cdot \mathbf{G} \cdot d \mathbf{W}_{t} .
$$

As in the previous section, equations (12) and (14) are just a way of rewriting the wavefunction dynamics. The notable feature of this decomposition is that the Hamilton-Jacobi equation (12) remains unchanged from its original form (3). Only the forward equation for the quantum probability distribution (14) includes additional stochastic terms. Note in particular that if we set $g=0$ in (14) we recover the forward equation (5).

There are at least two existing ways to interpret the GRW theory. One is to suppose that the wavefunction is representative of matter density in (threedimensional) space. For example, we could specifically define the matter density for constituent particle $i$ as

$$
\mathcal{M}_{i}(x ; t)=m_{i} \int d \mathbf{x} \delta\left(x_{i}-x\right)|\psi(\mathbf{x} ; t)|^{2},
$$

with the total matter density given by summing over the individual matter densities for each particle. With this definition, a superposition of two displaced bulk objects would correspond (at least temporarily before collapsing) to a matter distribution divided between the two locations. Following collapse the matter density would all be concentrated at one of the locations (this implies that matter density is not locally conserved). Another interpretation is to suppose that the collapse centers $z_{i}$ define a discrete (classical) image of the location of matter in space and time. Note that these 'hits' are concentrated where the quantum amplitude is greatest. The wavefunction is then an elaborate means of determining the likely distribution of discrete hits. (In moving to a continuous model the role of $z_{i}$ is replaced by the stochastic process $\left\{\langle\mathbf{x}\rangle_{t}+\mathbf{G}^{-1} \cdot\left(d \mathbf{W}_{t} / d t\right)\right\}$, see footnote 2)

Brownian motion by $d W_{i}=d W_{i}^{\prime}-g_{i}\left\langle x_{i}\right\rangle_{t} d t$. Including the contributions from all particles and normalizing the wavefunction leads to equation (11). A similar comparison between discrete and continuous models is made in Sec IV C. of [10]. 
In the next section we will develop our BM-GRW hybrid model. (Here the interpretation will be more clear cut: as with BM the model concerns the motion of a set of particles.) To do this we will have to understand the way in which the stochastic equation (14) can be thought of as the classical forward equation for a distribution of particles on configuration space (thus ensuring, once quantum equilibrium is assumed, that the statistical properties of the particles reproduce the statistical properties of the wavefunction). It will turn out that the particles satisfy exactly the same guiding equation as for BM (6). The stochasticity of the probability density on configuration space will be understood to result from an updating procedure based on a continuous stream of noisy information relating to the individual particle positions. The situation corresponds to the problem of nonlinear filtering.

\section{Nonlinear filtering}

In this section we explain the classical nonlinear filtering problem and its relevance to GRW theory. A more detailed introductory account of the nonlinear filtering problem can be found in [12].

All stochastic processes will be defined on a fixed probability space $(\Omega, \mathbb{P}, \mathcal{F})$ on which there is specified a filtration $\left\{\mathcal{F}_{t}\right\}$. We shall be concerned with some unobserved signal process $\left\{\mathbf{X}_{t}\right\}$ and a related (noisy) observation process $\left\{\mathbf{Y}_{t}\right\}$. The signal process $\left\{\mathbf{X}_{t}\right\}$, assumed to be adapted to $\mathcal{F}_{t}$, cannot be observed directly. Information about the signal process is obtained only from the observations $\left\{\mathbf{Y}_{t}\right\}$. Given some prior distribution for $\mathbf{X}_{0}$, the nonlinear filtering problem is simply to determine the distribution of $\mathbf{X}_{t}$ conditional on $\left\{\mathbf{Y}_{s} ; 0 \leq s \leq t\right\}$.

Specifically we will consider a version of the nonlinear filtering problem in which the observation process takes the form

$$
\mathbf{Y}_{t}=\int_{0}^{t} \mathbf{G} \cdot \mathbf{X}_{s} d s+\mathbf{B}_{t}
$$

where $\left(\mathbf{B}_{\mathbf{t}}, \mathcal{F}_{\mathbf{t}}\right)$ is a standard multivariate Brownian motion process and $\mathbf{G}$ is a real constant diagonal matrix; the signal process $\left\{\mathbf{X}_{t}\right\}$ takes the form

$$
\mathbf{X}_{t}=\mathbf{X}_{0}+\int_{0}^{t} \mathbf{F}\left(\mathbf{X}_{s}\right) d s
$$

where $\mathbf{F}$ is some vector-valued differentiable function of $\mathbf{X}_{t}$. For simplicity we assume that $\left\{\mathbf{X}_{t}\right\}$ and $\left\{\mathbf{Y}_{t}\right\}$ are a real-valued processes; for technical reasons we assume that $\mathbb{E}\left[\int_{0}^{t}\left|\mathbf{G} \cdot \mathbf{X}_{s}\right|^{2} d s\right]<\infty$, and that $\mathbf{X}_{s}$ and $\mathbf{B}_{s}$ are independent of $\mathbf{B}_{v}-\mathbf{B}_{u}$ for $s<u<v$ (this is used in the result of Fujisaki, Kallianpur, and Kunita [11, see below).

With $\mathcal{Y}_{t}:=\sigma\left\{\mathbf{Y}_{s} ; 0 \leq s \leq t\right\}$ (the $\sigma$-field generated by $\left\{\mathbf{Y}_{s} ; 0 \leq s \leq t\right\}$ ), our objective is to compute quantities of the form $\mathbb{E}\left[h\left(\mathbf{X}_{t}\right) \mid \mathcal{Y}_{t}\right]$, i.e. best estimates of functions $h$ of the signal at time $t$ conditional on the information contained in 
the noisy observations between times 0 and $t$. For any process $\eta_{t}$ we shall use the notation $\left\langle\eta_{t}\right\rangle:=\mathbb{E}\left[\eta_{t} \mid \mathcal{Y}_{t}\right]$.

Having presented the problem in mathematical terms we proceed by defining the innovations process,

$$
\mathbf{W}_{t}:=\mathbf{Y}_{t}-\int_{0}^{t} \mathbf{G} \cdot\left\langle\mathbf{X}_{s}\right\rangle d s
$$

It can be shown that $\left(\mathbf{W}_{t}, \mathcal{Y}_{t}\right)$ is a standard multivariate Brownian motion process as follows. From (18) and (16) we have

$$
\mathbb{E}\left[\mathbf{W}_{t} \mid \mathcal{Y}_{s}\right]=\mathbf{W}_{s}+\mathbb{E}\left[\int_{s}^{t} \mathbf{G} \cdot\left\{\mathbf{X}_{u}-\left\langle\mathbf{X}_{u}\right\rangle\right\} d u+\mathbf{B}_{t}-\mathbf{B}_{s} \mid \mathcal{Y}_{s}\right]
$$

Since $\mathbf{B}_{t}-\mathbf{B}_{s}$ is independent of $\mathcal{Y}_{s}$ and has zero expectation we find that $\left(\mathbf{W}_{t}, \mathcal{Y}_{t}\right)$ is a martingale: $\mathbb{E}\left[\mathbf{W}_{t} \mid \mathcal{Y}_{s}\right]=\mathbf{W}_{s}$. The quadratic variation of $\mathbf{W}_{t}$ must equal the quadratic variation in $\mathbf{B}_{t}$ (since the quadratic variation of $\int_{0}^{t} \mathbf{G}$. $\left\{\mathbf{X}_{s}-\left\langle\mathbf{X}_{s}\right\rangle\right\} d s$ is zero), therefore $\left(\mathbf{W}_{t}, \mathcal{Y}_{t}\right)$ must be a standard multivariate Brownian motion.

Now we introduce the result of Fujisaki, Kallianpur, and Kunita 11] that every square-integrable martingale $\left(m_{t}, \mathcal{Y}_{t}\right)$ has the representation

$$
m_{t}=\mathbb{E}\left[m_{0}\right]+\int_{0}^{t} \boldsymbol{\eta}_{s} \cdot d \mathbf{W}_{s},
$$

where $\int_{0}^{t} \mathbb{E}\left[\boldsymbol{\eta}_{s} \cdot \boldsymbol{\eta}_{s}\right] d s<\infty$ and $\left\{\boldsymbol{\eta}_{t}\right\}$ is adapted to $\mathcal{Y}_{t}$. With this result we will calculate $\left\langle h\left(\mathbf{X}_{t}\right)\right\rangle$. First consider a generic real-valued $\mathcal{F}_{t}$-measurable random process $\xi_{t}$ of the form

$$
\xi_{t}=\xi_{0}+\int_{0}^{t} \alpha_{s} d s
$$

with $\int_{0}^{t} \alpha_{s} d s$ of bounded variation. We define

$$
\mu_{t}:=\left\langle\xi_{t}\right\rangle-\left\langle\xi_{0}\right\rangle-\int_{0}^{t}\left\langle\alpha_{s}\right\rangle d s,
$$

and with $0<s<t$ we have

$$
\begin{aligned}
\mathbb{E}\left[\mu_{t} \mid \mathcal{Y}_{s}\right] & =\mu_{s}+\mathbb{E}\left[\left\langle\xi_{t}\right\rangle-\left\langle\xi_{s}\right\rangle-\int_{s}^{t}\left\langle\alpha_{u}\right\rangle d u \mid \mathcal{Y}_{s}\right] \\
& =\mu_{s}+\mathbb{E}\left[\xi_{t}-\xi_{s}-\int_{s}^{t}\left\langle\alpha_{u}\right\rangle d u \mid \mathcal{Y}_{s}\right] \\
& =\mu_{s}+\mathbb{E}\left[\int_{s}^{t}\left\{\alpha_{u}-\left\langle\alpha_{u}\right\rangle\right\} d u \mid \mathcal{Y}_{s}\right],
\end{aligned}
$$

which gives the result $\mathbb{E}\left[\mu_{t} \mid \mathcal{Y}_{s}\right]=\mu_{s}$, i.e. $\left(\mu_{t}, \mathcal{Y}_{t}\right)$ is a martingale. 
Next using (20) we can represent $\mu_{t}$ as a stochastic integral with respect to the innovations process. We have

$$
\begin{aligned}
\left\langle\xi_{t}\right\rangle & =\left\langle\xi_{0}\right\rangle+\int_{0}^{t}\left\langle\alpha_{s}\right\rangle d s+\mu_{t} \\
& =\left\langle\xi_{0}\right\rangle+\int_{0}^{t}\left\langle\alpha_{s}\right\rangle d s+\int_{0}^{t} \boldsymbol{\eta}_{s} \cdot d \mathbf{W}_{s}
\end{aligned}
$$

for some yet-to-be-determined process $\boldsymbol{\eta}_{t}$. To find $\boldsymbol{\eta}_{t}$ we use the result

$$
\mathbb{E}\left[\xi_{t} \mathbf{Y}_{t}-\left\langle\xi_{t}\right\rangle \mathbf{Y}_{t} \mid \mathcal{Y}_{s}\right]=\mathbb{E}\left[\xi_{t} \mathbf{Y}_{t}-\left\langle\xi_{t} \mathbf{Y}_{t}\right\rangle \mid \mathcal{Y}_{s}\right]=0,
$$

for $s<t$. From equations (16) and (21) we have

$$
\xi_{t} \mathbf{Y}_{t}=\xi_{0} \mathbf{Y}_{0}+\int_{0}^{t} \xi_{s}\left\{\mathbf{G} \cdot \mathbf{X}_{s} d s+d \mathbf{B}_{s}\right\}+\int_{0}^{t} \mathbf{Y}_{s} \alpha_{s} d s
$$

and from equations (18) and (24) we have

$$
\begin{aligned}
\left\langle\xi_{t}\right\rangle \mathbf{Y}_{t}=\left\langle\xi_{0}\right\rangle \mathbf{Y}_{0} & +\int_{0}^{t}\left\langle\xi_{s}\right\rangle\left\{\mathbf{G} \cdot\left\langle\mathbf{X}_{s}\right\rangle d s+d \mathbf{W}_{s}\right\} \\
& +\int_{0}^{t} \mathbf{Y}_{s}\left\{\left\langle\alpha_{s}\right\rangle d s+\boldsymbol{\eta}_{s} \cdot d \mathbf{W}_{s}\right\}+\int_{0}^{t} \boldsymbol{\eta}_{s} d s .
\end{aligned}
$$

Inserting these two expressions into (25) we find that $\boldsymbol{\eta}_{t}$ satisfies

$$
\boldsymbol{\eta}_{t}=\mathbf{G} \cdot\left\{\left\langle\xi_{t} \mathbf{X}_{t}\right\rangle-\left\langle\xi_{t}\right\rangle\left\langle\mathbf{X}_{t}\right\rangle\right\}
$$

and combining this result with equation (24) we have

$$
\left\langle\xi_{t}\right\rangle=\left\langle\xi_{0}\right\rangle+\int_{0}^{t}\left\langle\alpha_{s}\right\rangle d s+\int_{0}^{t}\left\{\left\langle\xi_{s} \mathbf{X}_{s}\right\rangle-\left\langle\xi_{s}\right\rangle\left\langle\mathbf{X}_{s}\right\rangle\right\} \cdot \mathbf{G} \cdot d \mathbf{W}_{s}
$$

This is the key result for our nonlinear filtering problem. This equation describes the dynamics of the best estimate of the random variable $\xi_{t}$ conditional on the noisy information $\mathcal{Y}_{t}$. Note that when $\xi_{t}$ takes the form $\xi_{t}=h\left(\mathbf{X}_{t}\right)$, the process $\alpha_{t}$ is given by $\alpha_{t}=\left.\mathbf{F}\left(\mathbf{X}_{t}\right) \cdot \boldsymbol{\nabla} h(\mathbf{x})\right|_{\mathbf{x}=\mathbf{X}_{t}}$.

More generally we can consider the probability distribution of $\mathbf{X}_{t}$ conditional on $\mathcal{Y}_{t}$ which we represent by $\rho(\mathbf{x} ; t)$. Given that $\mathbb{E}\left[h\left(\mathbf{X}_{t}\right) \mid \mathcal{Y}_{t}\right]=\int d \mathbf{x} h(\mathbf{x}) \rho(\mathbf{x} ; t)$, we can perform a straightforward integration by parts in equation (29) to derive the conditional forward equation

$$
d \rho(\mathbf{x} ; t)=-\boldsymbol{\nabla} \cdot\{\rho(\mathbf{x} ; t) \mathbf{F}(\mathbf{x})\} d t+\rho(\mathbf{x} ; t)\left\{\mathbf{x}-\left\langle\mathbf{X}_{t}\right\rangle\right\} \cdot \mathbf{G} \cdot d \mathbf{W}_{t} .
$$

This equation describes the time development of the probability distribution for $\mathbf{X}_{t}$ based on the fact that (i) $\mathbf{X}_{t}$ is a random process with dynamics described by (17); and that (ii) the probability density is continually updated with noisy information concerning $\mathbf{X}_{t}$ of the form (16). 
By now the similarity of (30) to (14) is clear. Once we interpret the signal process as the positions of a set of particles in space we can complete the picture by choosing $\mathbf{F}\left(\mathbf{X}_{t}\right)=\mathbf{M}^{-1} \cdot \nabla S\left(\mathbf{X}_{t} ; t\right)$ where $S$ satisfies the GRW equations (12) and (14) $\left(\mathbf{F}\left(\mathbf{X}_{t}\right)\right.$ as defined here will be $\mathcal{Y}_{t}$-previsible).

From (17) we then recover the guiding equation (6). Assuming that the quantum equilibrium hypothesis is satisfied at some initial time $t$ (whereby the probability distribution of particle positions on configuration space is equal to the GRW quantum probability distribution $|\psi|^{2}$ ), we find that the quantum expectation of $\mathbf{x}$ is the same as the conditional stochastic expectation of $\mathbf{X}_{t}$

$$
\langle\mathbf{x}\rangle_{t}=\int d \mathbf{x} \psi^{*}(\mathbf{x} ; t) \mathbf{x} \psi(\mathbf{x} ; t)=\int d \mathbf{x} \mathbf{x} \rho(\mathbf{x} ; t)=\left\langle\mathbf{X}_{t}\right\rangle .
$$

Finally taking the matrix $\mathbf{G}$ of this section to be equal to the matrix $\mathbf{G}$ defined in the previous section we have shown that (30) and (14) are equivalent. Through this equivalence we identify the $\mathbb{P}$-Brownian motion of the GRW equation (11) (which is related to the random localization centers) with the innovations process.

To summarize we can express the GRW theory in terms of a set of true particle positions satisfying a Bohmian particle guiding equation of the form (6) but dependent on an action $S$ determined from the GRW equations. Given an initial state of quantum equilibrium, the distribution of particle positions on configuration space will satisfy an equation precisely of the form (14) provided that we update the distribution with noisy information (16) concerning the precise whereabouts of the particles. This means that a state of quantum equilibrium will be sustained by the equations of motion.

We could at this stage claim to have rederived the equation for the wavefunction satisfied in GRW theory from the standard equations of BM by introducing this noisy information process. Starting with the system of equations (3) (5) (6), the additional noisy information forces us to replace (15) with (30). The result is the stochastic GRW equations for the wavefunction where the noisy information process seen as the source of the stochastic behavior.

\section{Discussion}

Having demonstrated that the BM-GRW theory is self-consistent, let us return to the question of why we would attempt to construct it in the first place. The first objective is simply to better understand the relationship between BM and GRW. From this point of view the BM-GRW theory acts as a stepping stone between the two. From BM-GRW we can either move to BM by removing the noisy information process and its effect on the wavefunction, or, move to GRW by dropping the hidden particle trajectories and regarding the wavefunction on its own. This helps to clarify the relationship between the two underlying theories. However, we will argue that the advantages of BM-GRW make it worth considering as a theory in its own right. 
A common criticism of BM is that, whereas the wavefunction has an influence on the set of particles, the particles have no influence over the wavefunction. Not only does this conflict with the universal principle for laws of physics stating that any action is matched by a reaction, it also leads to a lot of redundancy in the wavefunction. For every branch of the wavefunction containing the actual particle trajectories, there are countless other branches corresponding to every other potential 'world' which would have been realized had the particle positions been different. The effects of decoherence soon disable the influence of other branches on the particle trajectories, leaving much of the wavefunction redundant. Nonetheless these redundant branches are an essential element of $\mathrm{BM} 3$.

In the BM-GRW theory the particle positions do influence the wavefunction. This comes from identifying the noise in the GRW equations for the wavefunction with the innovations process which represents information about the signal process (the particle positions). The influence of particles on the wavefunction is unusual in the sense that it does not result from a direct interaction between particles and wavefunction (as can be said for the particle guiding equation). Rather it results from a transfer of information from particles to wavefunction. The outcome is that the complete wavefunction continually reflects the true particle trajectories and the redundant branches find themselves diminished.

This in turn implies that for the BM-GRW theory it is possible to adhere to a strict form of quantum equilibrium involving the complete wavefunction, even when describing processes such as quantum measurements. As discussed in the introduction, for BM, quantum equilibrium is only satisfied for an effective wavefunction. Although this is not necessarily a criticism, it does mean that a degree of judgment is required to determine what the effective wavefunction is. The standard procedure (see [16]) is to first divide the total configuration into subsystem and environment configurations $\mathbf{X}_{t}=\left(\mathbf{X}_{t}^{\mathrm{S}}, \mathbf{X}_{t}^{\mathrm{E}}\right)$. The effective wavefunction is then given by replacing the environment degrees of freedom in the complete wavefunction with their actual configurations: $\psi_{\mathrm{eff}}\left(\mathbf{x}^{S}\right)=\psi\left(\mathbf{x}^{S}, \mathbf{X}_{t}^{\mathrm{E}}\right)$. In a quantum measurement situation we can choose the macroscopic measuring device to be the environment. The dynamics of $\mathbf{X}_{t}^{\mathrm{E}}$, given by (6), typically mean that the effective wavefunction does not strictly satisfy a (subsystem) Schrödinger equation. In fact, the configuration of the measuring device (post measurement) will determine a collapse of the effective wavefunction.

In the BM-GRW theory the wavefunction actually collapses about the true particle trajectories. (In particular, bulk superpositions such as those involving macroscopic measuring devices are naturally suppressed by the wavefunction dynamics). The ambiguous procedure for determining the effective wavefunction is therefore unnecessary since the complete wavefunction $\psi$ is always representative (to the best of our knowledge) of the distribution of particles on

\footnotetext{
3 This criticism of BM has led several authors to argue that BM is little more than a version of the many-worlds interpretation in which the particle trajectories are a way to select one particular world. E.g., see [13], and for some counter arguments see [14]. It has also led Dürr, Goldstein, and Zanghì [15] to suggest that the wavefunction should be regarded as nomological, with a role analogous to the Hamiltonian in classical mechanics.
} 
configuration space.

Finally BM-GRW allows us to take a new perspective when interpreting the GRW theory. In BM we understand that the theory ultimately describes the behavior of a set of particles. Particles are taken to constitute the substance of the world. The wavefunction is seen as a real element of the theory but only insofar as it can influence the behavior of particles. We can carry this interpre-

tation over to the BM-GRW theory and also use it to justify equation (15) for the matter density in GRW.

\section{References}

[1] J. S. Bell, Speakable and unspeakable in quantum mechanics, Cambridge (2004).

[2] D. Bohm, Phys. Rev. 85, (1952) 166 \& 180.

[3] A. Bassi \& G.C. Ghirardi, Phys. Rept. 379, (2003) 257.

[4] P. Pearle, in: Open Systems and Measurement in Relativistic Quantum Field Theory, H.P. Breuer \& F. Petruccione eds., Springer Verlag (1999).

[5] G.C Ghirardi, A. Rimini, \& T. Weber, Phys. Rev. D34, (1986) 470.

[6] V. Allori et al., Brit. J. Phil. Sci., (2008) 353.

[7] D. Brody \& L. Hughston, J. Phys. A: Math. Gen. 39, (2006) 833.

[8] D. Brody, L. Hughston, \& A. Macrina, Int. J. Theor. Appl. Fin. 11, (2008) 107

[9] D. J. Bedingham, J. Phys. A: Math. Theor. 42, (2009) 465301.

[10] G.C. Ghirardi, P. Pearle, \& A. Rimini. Phys. Rev. A42, (1990) 78.

[11] M. Fujisaki, G. Kallianpur, \& H. Kunita, Osaka J. Math. 9, (1972) 19.

[12] M. Davis \& S. Marcus, in: Stochastic Systems: The mathematics of filtering and Identification and applications, M. Hazewinkel \& J. C. Willems eds., Reidel (1981).

[13] H. R. Brown \& D. Wallace, Found. Phys. 35, (2005), 517.

[14] A. Valentini, in: Many Worlds? Everett, Quantum Theory, and Reality, S. Saunders et al. eds., Oxford (2010).

[15] D. Dürr, S. Goldstein, \& N. Zanghì, in: Experimental Metaphysics: Quantum Mechanical Studies for Abner Shimony, Volume One, R. S. Cohen, M. Horne, \& J. Stachel eds., Springer (1997).

[16] D. Dürr, S. Goldstein, \& N. Zanghì, J. Stat. Phys. 67, (1992) 843. 